\title{
Current Situation and Countermeasures of Hypertension Health Management in Community
}

\author{
Zehui Jiang ${ }^{1}$, Jun Zhang ${ }^{2}$ \\ ${ }^{1}$ Jiangxi University of Traditional Chinese Medicine, Nanchang, Jiangxi, China; \\ ${ }^{2}$ Department of Cardiovascular Medicine, the 94th Hospital of Chinese People's Liberation Army, Nanchang, \\ Jiangxi, China \\ Communication Author: Jun Zhang
}

Keywords: Hypertension; health management; community

\begin{abstract}
Although the community health standard management of hypertension has been implemented for many years in China, it has achieved good results in reducing the incidence and mortality of cardiovascular and cerebrovascular diseases, but the implementation of specific methods and measures is not in place. Leading to hypertension "three high", "three low" phenomenon can not be fundamentally changed. This paper first analyzes the current situation of community hypertension health management, and then puts forward to the main problems of community hypertension health management, and finally puts forward to the countermeasures according to the problem.
\end{abstract}

Hypertension is an important pathogenic factor of stroke, coronary heart disease and other cardiovascular diseases, and it is extremely harmful to human health. It has become a major public health problem in worldwide. Hypertension has become one of the most important chronic diseases in the community public health service in china. How to do a good job in community hypertension health management has become a very important issue.

\section{Community hypertension health management present situation}

The comprehensive prevention and control measures of community hypertension in our country have been carried out earlier, and have achieved good results in reducing the incidence of cardiovascular and cerebrovascular diseases and mortality. The community hypertension health management mainly uses the method including the community hypertension comprehensive prevention and control, the hypertension three levels of management as well as hypertension standardization management at the present stage. However, due to the implementation of specific methods and measures are not in place, there is a lack of operability. Among them, the three level management of hypertension mainly refers to according to the blood pressure value of patients with hypertension, it is divided into a low-risk, medium risk, high risk, according to the patient's different conditions to take the corresponding treatment and management methods. However, the three level management of hypertension is passive management to a greater extent. If the patient's trust is low, it will seriously affect the patient's blood pressure control effect.

\section{The main problems of hypertension health management in community}

Government support and investment are low, affecting the process of community hypertension health management. At present, due to the less national investment in community health management, resulting in community public health managers work passively, seriously reducing the efficiency of community hypertension management. At the same time, due to the lack of community hypertension health management special funds, directly lead to community public health service institutions can not work normally, thus affecting the whole process of community hypertension health management. 
At present, the health resources of some large hospitals in China are far more than those in the community public health service organizations. Some community public health service institutions lack of resources and facilities are backward, which can not meet the requirements of community residents for primary medical services. At the same time, the community health service institutions which mainly manage chronic diseases and preventive health care are few, and the distribution is unreasonable, which leads to the community health management of hypertension become a form, not really implemented.

There are many people suffering from hypertension in China, but the number of community health workers is low, and their professional quality is low, which directly affects the efficiency of daily work. At present, most of the community doctors in China are transferred from specialist to general practitioner. Although the professional qualification of general practitioners, but the work concept and behavior is still at the level of professional doctors, and did not really integrate into the community residents health management jobs. At the same time, the community public health service doctors understanding of community health management of hypertension is not in place. They are pay attention to drug treatment, ignore the non drug treatment. At the same time, due to the low professional quality of medical staff in community public health services, most of the community residents are not willing to visit the hospital, which directly reduces the effect of community health management of hypertension.

\section{Community health management of hypertension Countermeasures}

The harm of hypertension on human health is not only a public health problem, but also a problem affecting social development, so it should be paid attention to. Adhere to the deepening of health system reform as the guide, and gradually establish and improve the government led, coordinated coordination of various departments of hypertension prevention and control coordination mechanism, improve community chronic disease prevention and control institutions. At the same time, we should build a scientific and reasonable prevention and control system of hypertension, and actively mobilize the masses to participate in it, so as to create a social environment conducive to the prevention and treatment of chronic diseases such as hypertension. Only in this way can the government community hypertension health management related policies and measures be implemented, and effectively improve the efficiency of prevention and treatment of chronic diseases such as hypertension.

In the process of social development, we should increase public health investment in accordance with the actual situation of social development, and give full play to the basic role of public finance in the prevention and control of chronic diseases such as hypertension. At the same time, we should establish a social multi-channel financing mechanism for the prevention and treatment of hypertension and other chronic diseases, encourage people from all walks of life to participate actively, and lay the foundation for improving the efficiency of prevention and control of chronic diseases such as hypertension in communities. At the same time, the government should ensure that the institution of community public health services in support of financial investment and allocation of resources, adhere to the different areas of public health resources rationally, and ensure the health service needs of residents to maintain consistency.

The important place of hypertension health management is doctors in community and community public health service station. They are important personnel of chronic disease management such as hypertension. However, the number of community health workers is low, and their professional quality is low, which directly affects the efficiency of daily work. Therefore, it is necessary to strengthen the training of personnel in community public health service institutions, pay attention to the training of general practitioners, and effectively improve the comprehensive business knowledge of community medical staff. Unified organization of community public health service personnel to participate in professional training, continuous introduction of more high-quality medical staff. At the same time, we should also establish a high quality general medical team, each team consists of 3 to 5 medical staff. These include general practitioners, nurses and administrators. And each general medical team should be effective management of community 
hypertension patients with chronic diseases, targeted implementation of health management methods, so as to promote the implementation of health management measures are truly implemented.

\section{Conclusion}

Community hypertension health management is a long-term, organized, planned systematic work. Only by deepening the reform of health system, improve community medical institutions; increase the government's public investment, expand social financing channels; strengthen cultivating talents, improve the overall quality of community medical staff, we can promote the community hypertension standardization management, and effectively control hypertension "three high" "three low" phenomenon, so as to ensure people's physical and mental health.

\section{References}

[1] Shen Xuying, Shi Ling, Wang Luchang. Effect evaluation of health management of general practitioner team in hypertension community [J]. China general practitioner 2010 (8):873 - 875.

[2] Liang Xiaohua, Gu Dongfeng. Cross-sectional investigation of community health management methods for hypertension in some areas of China [J]. Chinese Journal of health management, 2012 (1):53 - 56 .

[3] Tang Xinhua, Jin Hongyi, Xu Xiaoling, et al. Hypertension community "three management" model [J]. Chinese Journal of hypertension, 2009, 17 (1):71 - 75.

[4] Sun Lili, Niu Hui, Tao Mingchun. Problems faced by standardized management of high defect community [J]. Journal of community medicine, 2010, 8 (16):1 0 - 11.

[5] Zhu Mingfan, Luo Ningxiang, Zhu Yuping. The current situation of health resource allocation in China and the reform strategy [J]. general practice nursing, 2009, 1 (7):1 51 - 152 